\title{
Differential Equation of Hysteresis: Application to Partial Martensitic Transformation in Shape-Memory Alloys
}

\author{
A.A. Likhachev \\ Institute of Metal Physics, Academy of Sciences of the Ukraine, 36 Vernadsky street, 252142 Kiev, \\ Ukraine
}

\begin{abstract}
Because the martensitic transformations, as a rule, are first order transitions, a special attention should be attracted to a hysteretic behavior of shape-memory alloys. The most important characteristics of the temperatureor stress-induced martensitic transformation, have been studied in detail up to date. It has been shown that such macroscopic state variables as inelastic strain or volume fraction of the martensite are always complex multi-valued functions of the temperature and external stress.

Some phenomenological approaches for the thermomechanical state equations for shape memory alloys were recently published. In particular, a special type of differential equations describing evolution of the inelastic macroscopic strain and volume fraction of martensite as a functions of the temperature have been proposed in our recent papers [11 - 12]. These and some other problems associated with the irreversible processes caused by hysteresis will be discussed in the present paper.The main aim is to consider a simplest application of these equations to a strain evolution during the multiple temperature cycling in small temperature intervals.
\end{abstract}

\section{INIRODUCTION}

Many unusual thermomechanical properties of shape-memory alloys are directly connected with martensitic type phase transitions in these systems. Because the martensitic transformations, as a rule, are first order transitions, a special attention should be attracted to a hysteretic behavior of shape-memory alloys. The most important characteristics of the temperature- or stress-induced martensitic transformation, have been studied in detail in [1-5]. It has been shown that such macroscopic state variables as inelastic strain or volume fraction of the martensite are always complex multi-valued functions of the temperature and external stress. Therefore, the shape-memory alloys should be considered as systems having an infinite number of state equations, representing inelastic strain and volume fraction of martensite as a functions of the external stress and temperature,correspondingly.

Some of the phenomenological approaches for the thermomechanical state equations for shape memory alloys were recently published in [6-10,14]. In particular, a special type of differential equations describing evolution of the inelastic macroscopic strain and volume fraction of martensite as a functions of the temperature have been proposed in our recent papers [11-12]. Its application to a 
partial temperature cycling processes in shape-memory alloys and some other problems associated with the irreversible processes caused by hysteresis will be discussed in the present paper.

\section{STRATN EVOLUTION EQUATION}

In solids which do not undergo any structure transitions or plastic deformations the strain is known to be a single-valued function of stress and temperature. Controversially, in systems undergoing martensitic type of structure transformations the macroscopic strain will not be more a function of stress and temperature, but it becomes a function of the process of its changes. The typical temperature induced strain change processes at constant load condition for NiTialloy are indicated in Fig. 1 .

As follows from the results represented in Fig.1, under the external stress applied the temperature induced macroscopic strain change is characterized by the main hysteresis loop $\varepsilon=z_{-}(T)$ and $\varepsilon=z_{+}(T)$ corresponding to cooling an heating processes, respectively. Besides, a set of sub-loops representing partial transformation processes are also possible as shown here. The most general example of the strain change process is indicated in Fig.2. Therefore, in general, the macroscopic strain is always a definite function of the temperature change process and can take arbitrary values inside of the main hysteresis loop.

To describe the irreversible evolution of macroscopic variables caused by hysteresis the one possible way based on the differential equation method has been proposed in our works $[11,12]$. Such a possibility follows immediately from the fact that only a single path from each family in Fig.1 can pass trough a given point with coordinates $(\varepsilon, T)$ inside of the main hysteresis loop. Therefore, only a single value of the first derivative $d E / d T$ for the cooling or heating family of paths may correspond to each point in $\varepsilon-T$ plane. Mathematically this denotes that de/dT must be a single-valued function of $\varepsilon$ and $T$ separately for cooling and heating processes. According to these works each thermodynamical path belonging to cooling or heating family must satiafy the following type of differential equations:

$$
\mathrm{d} \varepsilon / \mathrm{dT}=\mathrm{S}_{ \pm}(\varepsilon, \mathrm{T})
$$

where $S_{ \pm}(\varepsilon, T)$ are single-valued functions of $\varepsilon, T$ corresponding to heatine and cooling process, respectively.

It has been also found that $S_{ \pm}(\varepsilon, T)$ can be expressed as a linear function of $\varepsilon$ :

$$
S_{ \pm}(\varepsilon, T)=\alpha_{ \pm}(T) \varepsilon+\beta_{ \pm}(T)
$$

with the temperature dependent coefficient $\alpha_{ \pm}(T)$ and $\beta_{ \pm}(T)$ depending only on the main hysteresis loop shape represented by $z_{ \pm}(T)$ functions :

$$
a_{ \pm}(T)= \pm \frac{d z \pm}{d T} \frac{1}{z_{+}-z_{-}} ; \quad \beta_{ \pm}(T)=\mp \frac{d z \pm}{d T} \frac{z \mp}{z_{+}-z_{-}}
$$

Therefore, the differential equations for basic heating and cooling paths family:

$$
d \varepsilon_{ \pm} / \mathrm{dT}= \pm \frac{\mathrm{dz} \pm}{\mathrm{dT}}\left(\frac{\varepsilon \pm-z^{\mp}}{z_{+}-z_{-}}\right)
$$

These equations give the possibility to predict the macroscopic strain evolution for any temperature change process. In accordance with Eq.(4) for this aim one should know only the information about the temperature behavior of $z_{ \pm}(\mathrm{T})$-paths representing the main hysteresis loop. 
In the present paper, by using the above-mentioned strain evolution equation and experimental $\mathbf{z}_{ \pm}(\mathrm{T})$-data for $\mathrm{Ni}-\mathrm{T} 1$ alloy studied in [12], the macroscopic strain behavior during the multi-fold cycling in a fixed temperature interval will be quantitatively calculated and briefly analyzed.

\section{APPLICATION TO CYCLING IN A SMALL TKMPGRATURR INTERVAL}

Consider a simplest application of these equations to a strain evolution during the temperature cycling in small temperature intervals studied recently by Humbeek at al. in [13]. These authors have classified the hysteresis sub-loops behavior during cycling in different small temperature intervals. The main result they observed is a subsequent drift (called by authors as creep-effect) of sub-loops induced by cycling and its stabilization after many cycles. In particular, positive as well as negative drift is possible. The positive drift is usually observed if cycling starts from the cooling branch of main hysteresis loop. If only a cycling process starts from the heating branch of loop then a negative sub-loops drift is observed.

Our equations give a natural way for a quantitative analysis of this problem. In particular, for very close temperatures: $T^{*}=T$ and $T^{*}=T+\delta T$ with $\delta T \rightarrow 0$, if we denote the strain value at $T^{\prime}=T$ after $n$-fold cycling between $T^{\prime}$ and $T^{\prime \prime}$ as $\varepsilon_{n}$, we can find the next strain value $\varepsilon_{n+1}$ by integration of Eq. (1) as follows:

$$
\varepsilon_{n+1}-\varepsilon_{n}=\left(S_{+}\left(\varepsilon_{n}, T\right)-S_{-}\left(\varepsilon_{n}, T\right)\right) \mathcal{E} T
$$

Taking into account Eqns. $(2,3)$ and solving this recurrence one can easily find the following results:

i). The only stabilized sub-loop corresponding to cycling between two fixed temperatures $\mathrm{T}^{\prime}$ and $\mathrm{T}^{\prime}$ ' exists inside of the main hysteresis $100 \mathrm{p}$.

ii) The evolution of strain value $\varepsilon_{n}$ after $n$-fold cycling can be represented as follows:

$$
\varepsilon_{n}=\left(\varepsilon^{*}-\left(\varepsilon_{0}-\varepsilon^{*}\right) \exp \left(-n / n^{*}\right)\right.
$$

where $\varepsilon^{*}(T)$ is the stabilization level of the strain in the asymptotic limit $n \rightarrow \infty$, $n^{*}(T)$ is the characteristic number of cycles required to achieve the stabilization level, $\varepsilon_{0}$ is the strain value before cycling.

iii) For very close temperatures $T^{\prime \prime}=T$ and $T^{\prime \prime}=T+\delta T$ with $\delta T^{*} \rightarrow 0$ the strain stabilization level $\varepsilon^{*}$ and characteristic number of cycles $n^{*}$ can be represented as follows:

$$
\begin{gathered}
E^{*}(T)=z_{+}(T) \frac{z_{-}^{\prime}(T)}{z_{+}^{\prime}(T)+z_{-}^{\prime}(T)}+z_{-}(T) \frac{z_{+}^{\prime}(T)}{z_{+}^{\prime}(T)+z_{-}^{\prime}(T)} \\
n^{*}(T)=\left(\frac{z_{+}^{\prime}(T)+z_{-}^{\prime}(T)}{z_{+}(T)-z_{-}(T)} \delta T\right)^{-1}
\end{gathered}
$$

Here, $z_{ \pm}(T)$ are the functions representing the main hysteresis loop and $z_{ \pm}^{\prime}(T)=$ $\mathrm{d} z_{ \pm}(\mathrm{T}) / \mathrm{AT}, \delta \mathrm{T}$ is the temperature cycling interval.

In the case that a cycling interval is not very small, we have calculated a set 


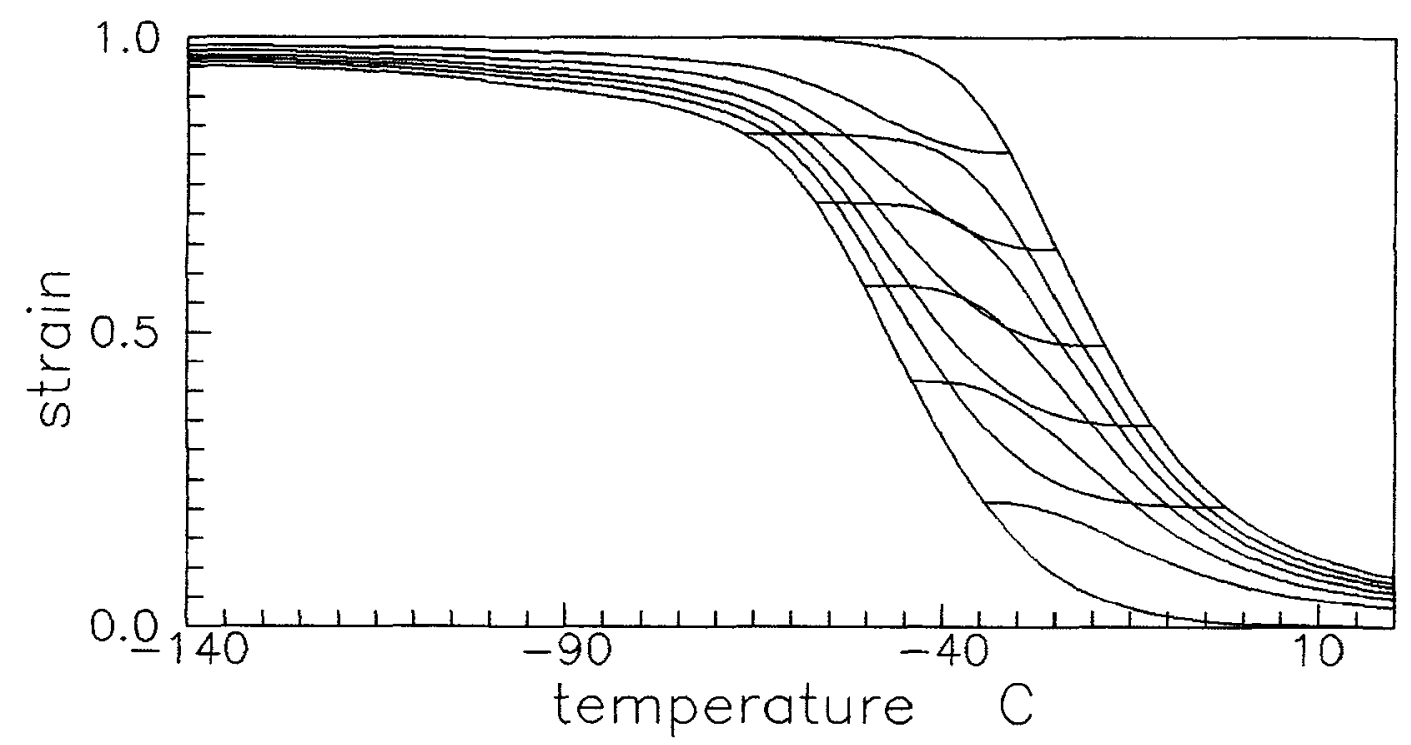

Fig.1. Main hysteresis loop and partial transformation paths representing strain-temperature behavior of NisiTi4a shape-memory alloy studied in [12].

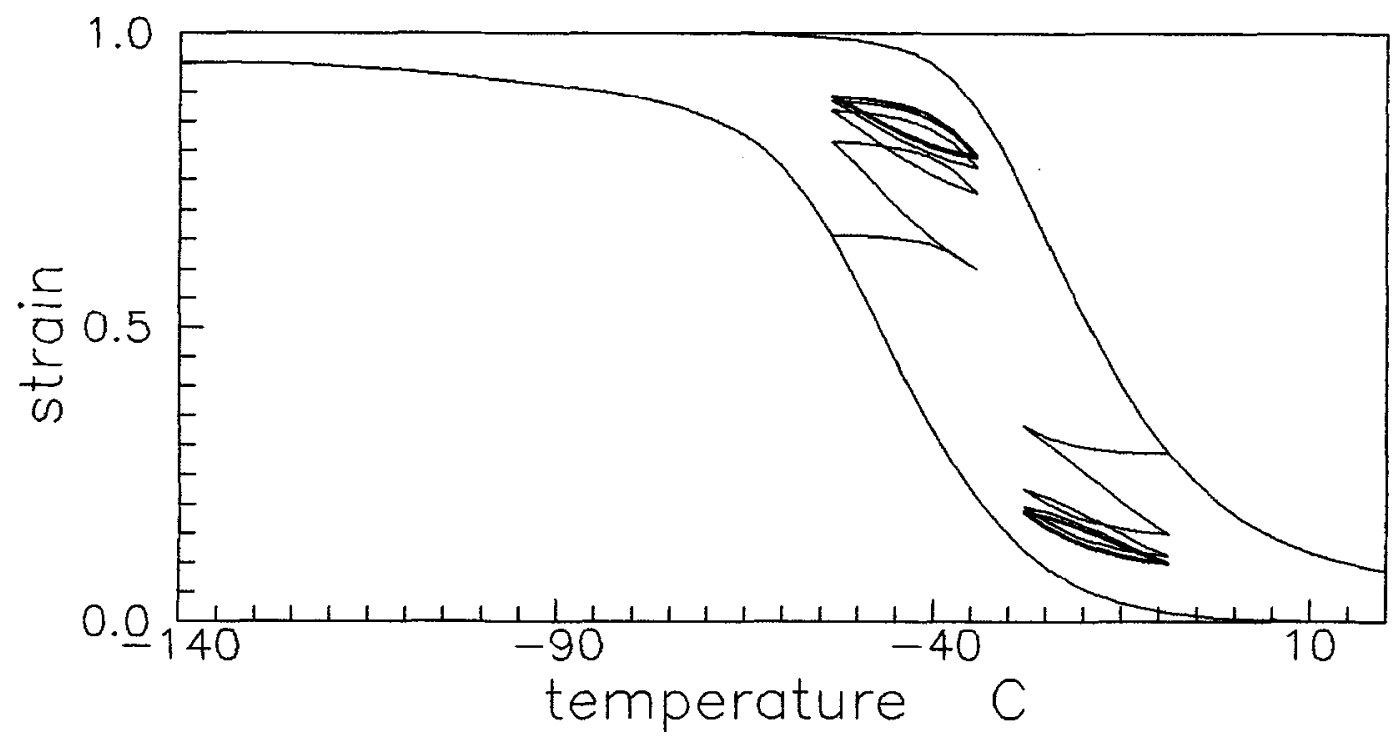

Fig.2. Main hysteresis loop and strain evolution paths corresponding to multi-fold cycling in small temperature intervals calculated according to present theory. 
of examples representing sub-10op dynamics during the temperature cycling at different temperatures by using the corresponding computer simulation procedure. These results are shown in Fig. 2 . Here we used the data representing main hysteresis loop of NiTi alloy. As seen from Fig.2 our results show the same behavior as in the above-mentioned experimental studies including a positive and negative sub-loops drift and its stabilization effect.

\section{References}

1. I.Cornelis, and C.M.Wayman, Scripta Met., 10,(1967), p. 359 .

2. K.Otsuka, C.M.Wayman, K.Nakai, H.Sakamoto, and K.Shimizu, Acta Met., 24, (1979),p.207.

3. L.Delaey, J.Van Humbeeck, M.Chandrasekharan, J.Janssen, M.Andrade, and N.Mwamba, Metals Forum, 4,(1981), p.164.

4. Yu.I.Paskal, and L.A.Monasevich, Fiz. Met. e Metallov. 52, p.1011, (1981), and Phys. Met. Metall. 52,(1981), p.95.

5. H.Verguts, L.Delaey, E.Aernoudt, and Vermeersch, (1983), in EUROMECH Colloquium, Warshawa, Poland, p.171.

6. J.S.Cory, and McNichols, J.Appl.Phys., 58,(1985), p.3282 .

7. E.Aernoudt, and Lu Li, (1986), in Proceed. Int. Symp. on Shape Memory, Guilin, China, p.23.

8. L.Delaey, and E.Aernoudt, (1986), in Proceed. of ICOMAT-86, Nara, Japan, p.926.

9. I.D.Mayergoyz, J. Appl. Phys., 57, (1985), p.3803.

10.P.Guelin, and D. Favier, J. de Mechanique 19, (1980), p.217 .

11.Yu.N.Koval, and A.A.Likhachev, Metallofizika, 5, (1988),p.28 .

12.A.A.Likhachev and Yu.N.Koval, Scr. Met. et Mat., 27, (1992),p.223

13.J.Van Humbeeck and R. Stalmans, Mat. Sci. Forum, 56-58, (1990), p.405.

14.J.Ortin, J. Appl. Phys., 71, (1992),p.1454. 